\title{
A Study of Active Contour Segmentation Models based on Automatic Initial Contour
}

\author{
Caibo $^{1,2 *}$, Zhigui Liu ${ }^{1,2}$, Junbo Wang ${ }^{1,2}$ and Yuyu Zhu ${ }^{1}$ \\ ${ }^{1}$ Southwest University of Science \& Technology, Mianyang Sichuan 621010, \\ China \\ ${ }^{2}$ China Academy of Engineering Physics, Mianyang, Sichuan 621000, China \\ caibo_bupt@126.com
}

\begin{abstract}
Image segmentation is a fundamental and challenging problem in image processing and often a vital step for high level analysis. Due to the active contour model (ACM) need to choose the initial contour for the following evolution, it limited the utilities of this kind of segmentation to a large extent. For the purpose of avoiding the processing of human choosing initial contour, in this paper, we proposed an automatic initial contour choosing algorithm of the input image information. Based on the chosen initial contour, the iterative efficient and the accuracy of segmentation have been improved when the initial contour is incorporated into the local based segmentation framework. Extensive experiments on synthetic and real images are provided to evaluate our method, showing significant improvements on the segmentation accuracy and stability, comparing to the human chosen initial contour, such as LBF and LGIF.
\end{abstract}

Keywords: Image segmentation, ACM model, Curve evolution, Initial contour, LBF, $L G I F$

\section{Introduction}

Active Contour Model (ACM) based segmentation algorithm has been widely investigated and applied to the image segmentation [1-16]. In general, the basic idea of active contour model is to deform an initial contour toward the actual boundary of the object. It may be categorized into edge-based and region based models. In edge and region based ACM, image gradient and statistical information are often used to stop the contours respectively. The benefit of this kind of models is that the image has no global constraints, thus the objective and the background can be heterogeneous and the final segmentation can be achieved easily. However, it relies heavily on edge information of the input image, when an edge of the region is weak, such as blurred, broken, the method may loss its roll.

Region-based active contour model (ACM) utilizes the objective and the background regions statistically and finds an energy optimum where the model best fits the image. Because of more advantages over edge-based ACMs, such as robustness for image with weak edges or without edges and insensitivity to the location of initial contours, region-based ACMs have been applied more popularly, in which, the Chan-Vese (CV) model is one of the most popular region-based models. However, techniques that attempt to model regions using these kinds of methods are usually not ideal for segmenting heterogeneous objects, or transitional regions, which frequently occur in natural images.

There are many methods in the literature which are aimed at improving the segmentation accurate by introducing more edge or region information into the active contour model. Because of the minimization of the energy function heavily depends on the gray scale, the distribution and area of the image regions, the total 
information often lead to the false segmentation in the local region of the image. Comparing to the different wrong segmentation of different models, the position of different initial contours may be the main drawbacks in natural image segmentation utilities. The accurate position of initial contour can drastically reduce the time needed for segmentation. In most of the papers, the position of the initial contour is set manually.

In this paper, we focus on the construction of initial contour in the evolution of active contour model. For the purpose of letting the let the initial contour more conform to the region and edge information of the different input images, we use the gradient of the region edges as the guided initial contour, and at the same time, the region information and local/global contrast are used to control the accuracy of the initial contour. The main advantages of our segmentation method can be highlighted as:

(1) In this paper we proposed a new algorithm to choose the initial contour which may improve the efficient of evolution to a large extent;

(2) Due to the initial contour is chosen according to the contrast and edge information of the different input images, this makes the initial contour more adaptable to the input image characters;

(3) Because of the proposed algorithm need not the need the human intervening, it makes the construction of automatic segmentation system more easily.

The remainder of this paper is organized as follows. In Section 2, we give a briefly review of the relative initial contour set algorithms. The proposed model is described in Section 3. Section 4 is the comparison of the proposed initial contour algorithm with different initial contour. We go on analyzing the results of the efficient and the convergence of each evolution in Section 5. Finally, the conclusion and limitations of our model have been discussed in Section 6.

\section{The Review and Discussion of the Related Works}

In this section, we give a review of the problem of initial contour in active contour based models. For a given image $I(x)$ on the image domain $R$, Chan-Vese [16] proposed to minimize the following energy equation:

$$
E^{C V}\left(c_{1}, c_{2}, C\right)=\lambda_{1} \int_{i n(C)}\left|I(x)-c_{1}\right|^{2} d x+\lambda_{2} \int_{o u t(C)}\left|I(x)-c_{2}\right|^{2} d x
$$

where $c_{1}$ and $c_{2}$ are two constants that approximate the average intensity inside and outside the curve, respectively. The coefficients $\lambda_{1}$ and $\lambda_{2}$ are fixed parameters.

By adding the regularizing term, such as the length of the contour and the area inside and outside the contour, the energy $E^{C V}\left(c_{1}, c_{2}, C\right)$ is defined as :

$$
\begin{aligned}
E^{C V}\left(c_{1}, c_{2}, C\right) & =\lambda_{1} \int_{\text {in }(C)}\left|I(x)-c_{1}\right|^{2} d x+\lambda_{2} \int_{\text {out }(C)}\left|I(x)-c_{2}\right|^{2} d x \\
& +\mu \text { Length }(C)+v \text { Area }(\text { in }(C))
\end{aligned}
$$

Using the level set to represent $C$, that is, $C$ is the zero level set of Lipschitz function $\phi(x)$, the energy function may be rewritten as:

$$
\begin{aligned}
E^{c V}\left(c_{1}, c_{2}, \phi\right) & =\lambda_{1} \int_{R}\left|I(x)-c_{1}\right|^{2} H(\phi(x)) d x+\lambda_{2} \int_{R}\left|I(x)-c_{2}\right|^{2}(1-H(\phi(x))) d x \\
& +\mu \int_{R} \delta(\phi(x))|\nabla \phi(x)| d x+v \int_{R} H(\phi(x)) d x
\end{aligned}
$$

where $H(\phi)$ and $\delta(\phi)$ are Heaviside function and Dirac function, respectively. The coefficients $\mu$ and $v$ are fixed parameters. 
The CV model has a good performance on image segmentation due to its ability of obtaining a larger convergence range and being less sensitive to the initialization. However, the CV model is only adapted for 2-phase image. If the intensities with inside $C$ or outside $C$ are not homogeneous, the constants $c_{1}$ and $c_{2}$ will not be accurate.

Following this way, there are some papers aimed at dividing the local and global characters of the image $[5,7-9,12]$, and constructing the energy function to solve the global and local problems. However, the edges are often exists in the different phase of image regions, and make the edges inhomogeneous in local regions. Simply sum the difference of all local regions may cause the global optimizing result inconsistence to the local dividing results. On the other hand, only using the local dividing result to approximate the global segmentation may cause the wrong segment of the local regions. Besides, the initial contour decided the initial evolution condition, if the initial contour is wrongly chosen, the evolution may lead to the wrong segmentation.

Less paper have given the analysis of the initial contour, in [17], Lingling Fang etc., give a dip of this problem. In this paper, they modeled the whole image by a Gaussian mixture distribution, and then use the mean and standard deviation describe the objective and background, respectively. The probability of observing the current pixel is

$$
p(I(x) \mid \Theta)=\sum_{i=1}^{2} \pi_{i} p_{i}\left(I(x) \mid \theta_{i}\right)
$$

where $I(x)$ is the intensity on the image domain, $i$ is the number of distributions $i=1,2, \Theta=\left(\pi_{1}, \pi_{2}, \theta_{1}, \theta_{2}\right)$ such that $\sum_{i=1}^{2} \pi_{i}=1$ and each $p_{i}$ is a density function parameterized by $\theta_{i}=\left(u_{i}, \sigma_{i}\right)$, the probability of a pixel $I(x)$ belonging to some region characterized by $\theta$ is

$$
p\left(I(x) \mid \theta_{i}\right)=\frac{1}{\sqrt{2 \pi \sigma_{i}^{2}}} \exp \left[\frac{-\left(I(x)-u_{i}\right)^{2}}{2 \sigma_{i}^{2}}\right]
$$

After the fitting of the image histogram, they use Bayes estimation to get a big or small state of each pixel $I(x)$, where the big state is used for denoting the objective and the small state is used for denoting the background.

On this condition, when the image is two-phased distribution, it may easily get the foreground and decide the initial contour. But, when the gray of the image region is transitional or the histogram of the image is not close to two Gaussian distribution, it may lose its role. Figure 1 shows the two kinds of image histogram fitting curve using the particle swarm optimization (PSO).

From Figure 1, we may find when the image histogram is close to 2-Gaussian distribution, the fitting curve have less difference than the other kinds of histogram. According to our experiments, when the initial contour is close to the region edges, the efficient and accurate of evolution would be dramatically improved. Along with the condition, the proposed algorithm would be proposed in the following section. 


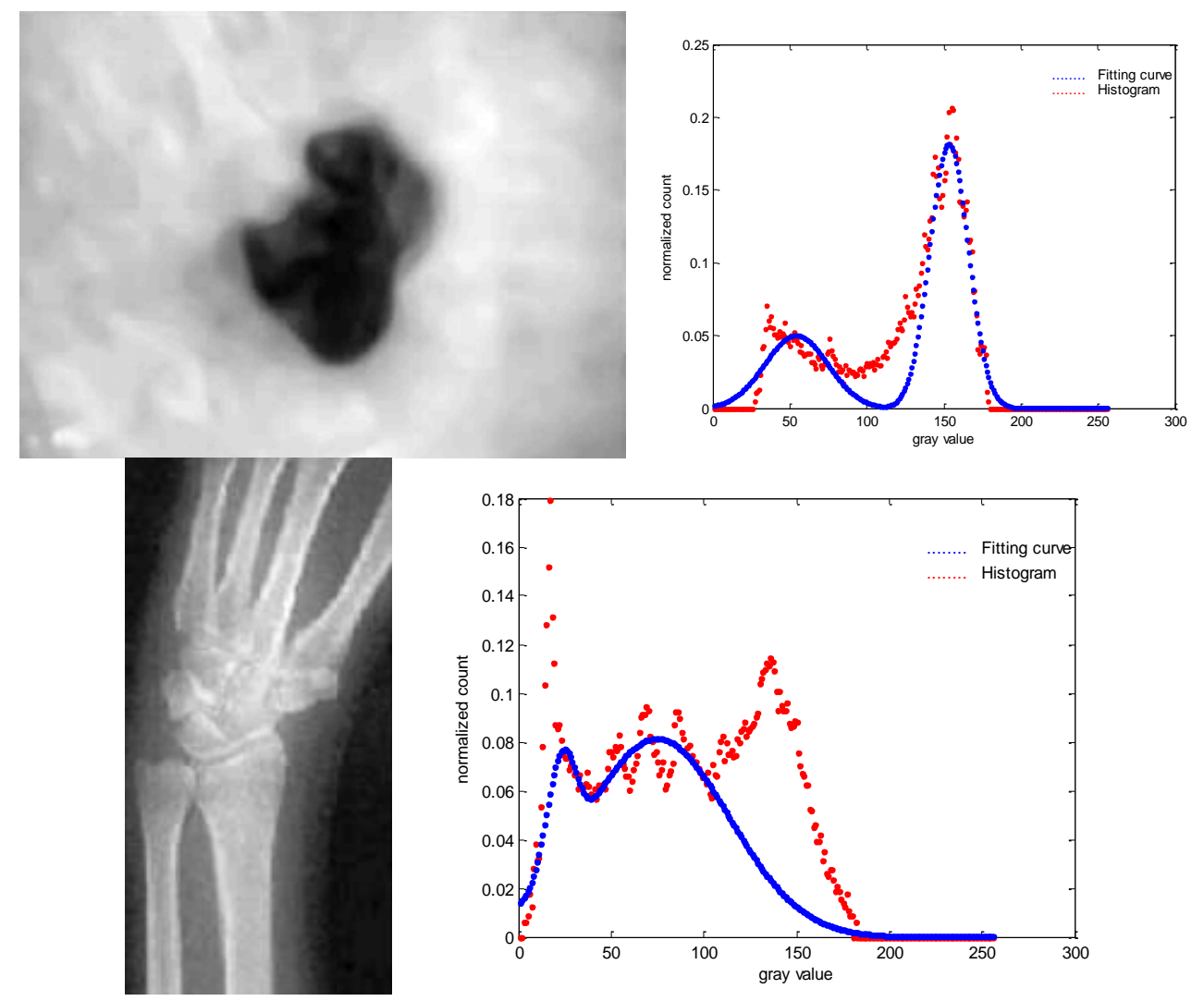

Figure 1. The Rough Image and the 2-Gaussian Mixture Model Fitting Result

\section{The Proposed Model}

\subsection{The Criteria of Initial Contour Choosing}

In this section, we will give the algorithm of the initial contour choosing. Before the construction of our model, we give an analysis of Haralick and Shapiro[18] proposed four criterial of image segmentation. In [18], a good segmentation criteria is defined as:

(1) Regions should be uniform and homogeneous with respect to some characteristic(s);

(2) Adjacent regions should have significant differences with respect to the characteristic on which they are uniform;

(3) Region interiors should be simple and without holes;

(4) Boundaries should be simple, not ragged, and be spatially accurate.

The first two criteria may be seen as the Characteristic Criteria, and the last two criteria are based on how likely each region is regarded as a single object by people, so it may be called Semantic Criteria. In active contour model, the choosing of initial contour may be seen as a pre-segmentation processing, so the initial contour should conform to the above mentioned criteria. Comparing to the characterizations of the image, the gradient and contrast may be the most important characters. Based on these two features, we define the criteria of initial contour choosing as

(a) The edge of the initial contour should cover or close to the image edges.

(b) The gray value of the initial contour should be homogeneous and high contrast. 


\subsection{The Choosing Algorithm of the Initial Contour}

According to the experiments of different images [17], we found the 2-Gaussian mixture model and ACM model have the similar convergence center, the upside and downside gray mean $c_{1} \approx u_{1}$ and $c_{2} \approx u_{2}$. And at the same time, the dividing of the image according to the mean gray value plays the same role. For the purpose of saving the timeconsuming of our algorithm, we use the mean value to divide the input image into different parts of gray distribution as the binary divisive procedure. After $N$ times dividing, the image is divided into $2^{N}$ parts in gray scale, the total number is $2^{N+1}-2$. Figure 2 shows the dividing of the rough image when $N=2$.

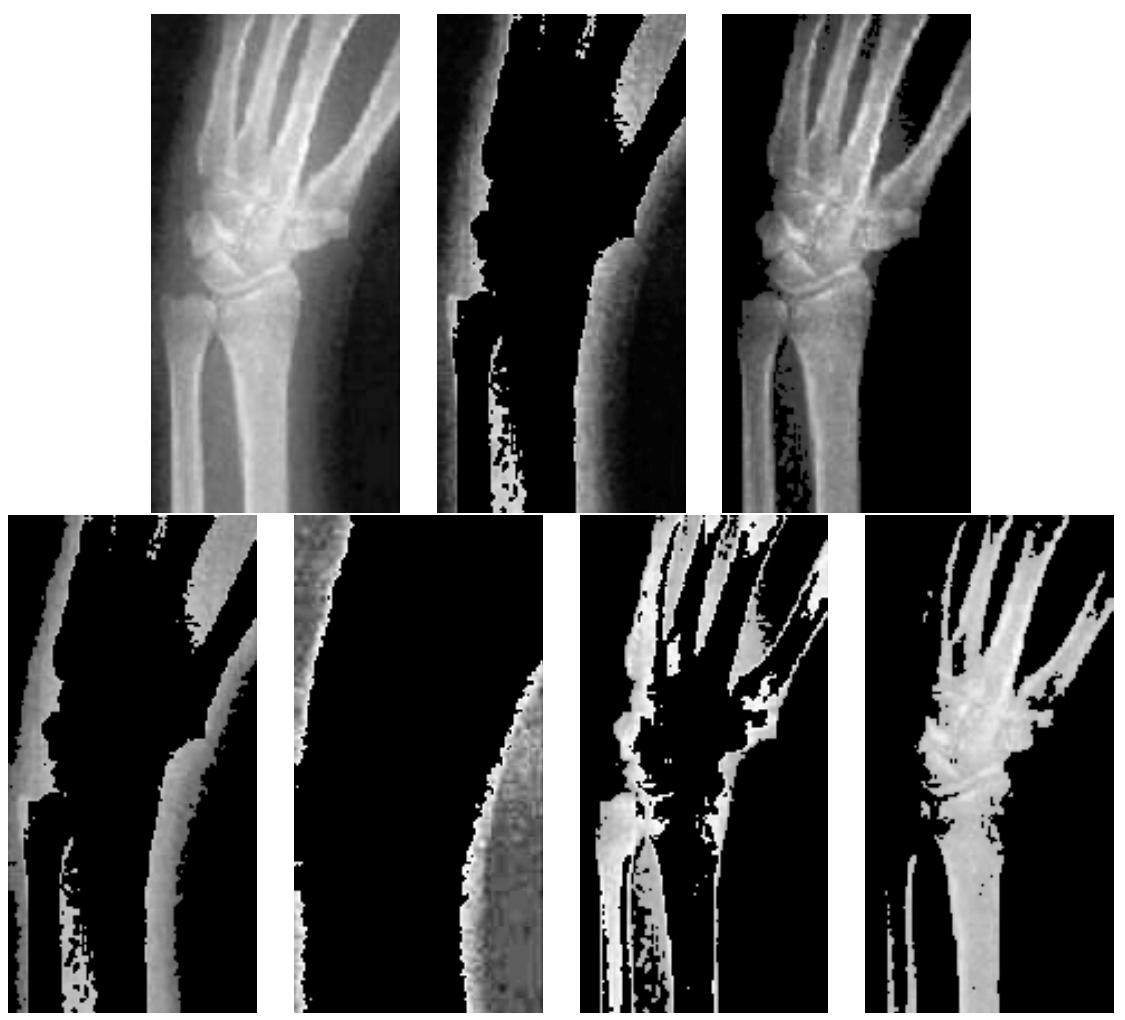

Figure 2. The Dividing of the Image, the First Row is the Rough Image First Dividing Results, and the Second Row is the Second Dividing Results

The dividing of the image as the first step of our algorithm is used to protect the homogeneous of the initial contour. The following step should keep the initial contour conform to the edges of the image region. In the detection of the image edges, there are many kinds of algorithm or operators, such as, the simple Gradient Vector function, the normal Gradient Modulus function, the Wavelet Modulus function, the Sobel operator, the Prewitt operator etc. Comparing to the Sobel and Prewitt operator, the gradient detector often get the double edges. So, in this paper, we choose the Sobel operator as the edge detector, and use the detect result as the reference of our initial contour choosing algorithm. 

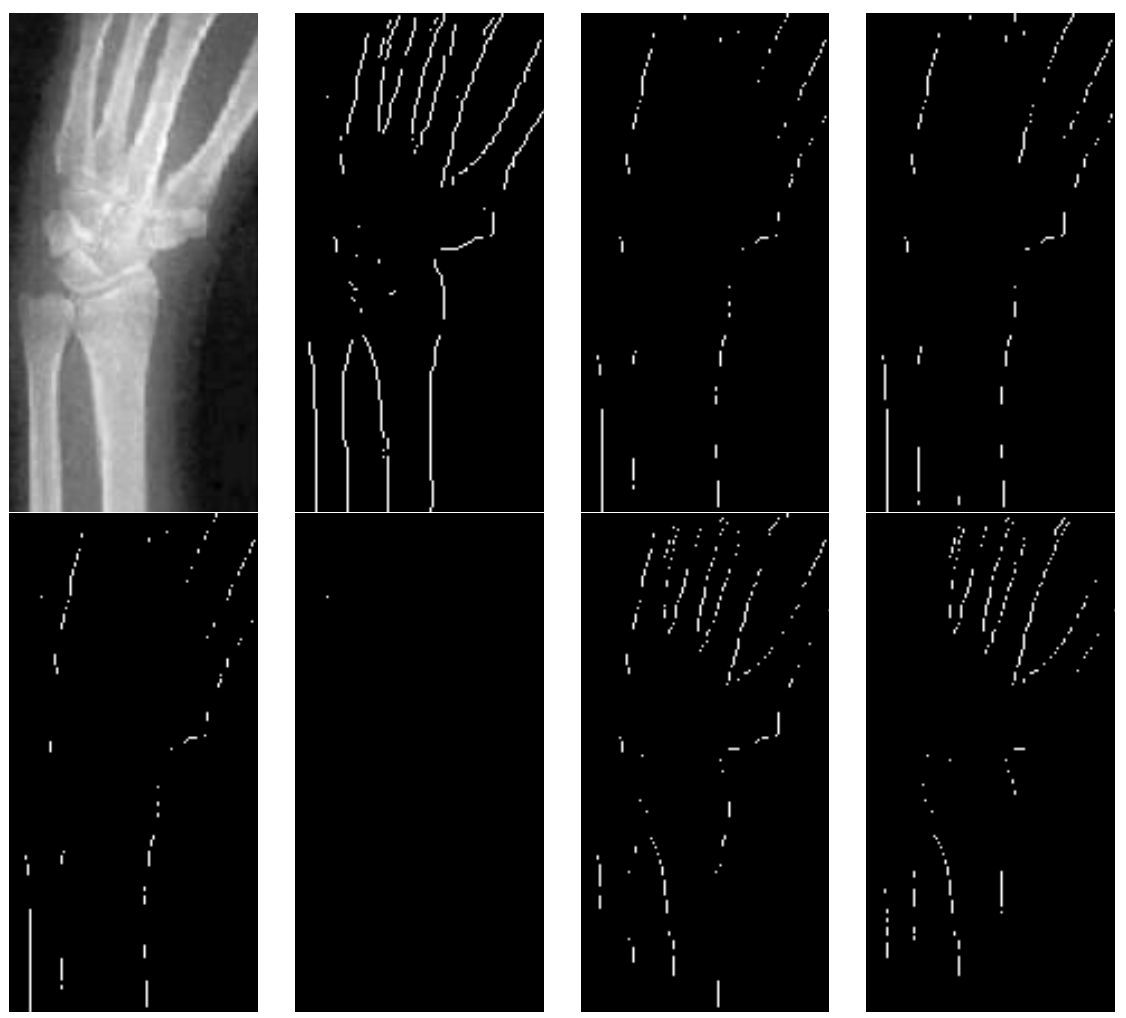

\section{Figure 3. The Edge of the Rough Image and the Covered Edge of Figure 2, the Divided Parts}

Figure 2 shows the covered edges of Sobel operator detecting results of Figure 2. If we choose the cover count as the standard of initial contour, the hole and region inner standard would be omitted. Because of the hole number is related to the edge number of the divided images, we choose the cover ratio of the edge points as the initial contour choosing standard.

For a given image $I$, the divided parts of the image constructed an image sequence $I_{i}, i=1,2, \cdots, 2^{N}-2$, where $N$ is the dividing times. The choosing which image $I_{j}$ of the initial contour should belong to is defined as

$$
\begin{aligned}
& R_{j}^{G}=\max \left\{R_{i}=\frac{S\left(E(I) \cap E\left(I_{i}\right)\right)}{S(E(I))}, i=1,2, \cdots, 2^{N}-2\right\} \\
& R_{k}^{L}=\max \left\{R_{i}=\frac{S\left(E(I) \cap E\left(I_{i}\right)\right)}{S\left(E\left(I_{i}\right)\right)}, i=1,2, \cdots, 2^{N}-2\right\}
\end{aligned}
$$

where $R_{k}^{L}$ and $R_{j}^{G}$ is the ratio of the current divided edges compared to the local and total image edges, $E(I)$ is the edge of the rough image, $E\left(I_{i}\right)$ is the edge of the i-th divided image, $S(\square)$ is the summation formula. When these two kinds of maximum ratio images have been gotten, we compare the region number of the two images, and choose the less one.

After the divided image has been chosen, the next problem is the choosing of the main regions as the initial contour. When the chosen image is concentrate, we may just use the total image as the initial contour. On the contrary, when the regions are dispersion, directly choosing of the total divided image may cause wrong segmentation in the 
following evolution. It is very difficult to choose the regions, especially when the input image region is multi-phased. For the purpose of making our proposed algorithm to realize the automatic choosing of initial contour, we only eliminate the little region of the current selected part $I_{i}$. Figure 4 shows the procedure of the proposed algorithm of automatic initial contour choosing.

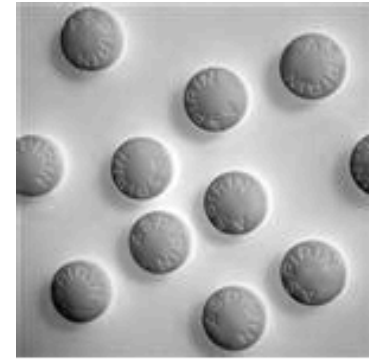

Rough image

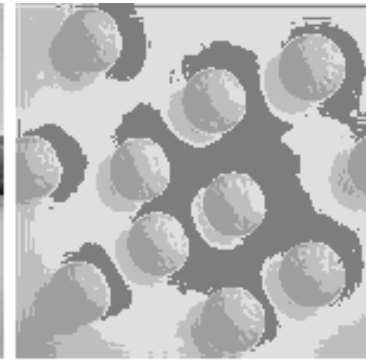

Divided results

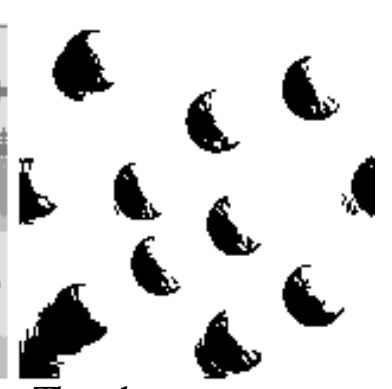

The chosen contour

Figure 4. The Proposed Algorithm of Initial Contour

\section{Implementation and Experimental Results}

In this section, we will focus on the role of the proposed algorithm in active contour based model. Before the experiments, we will give analysis of the different models, and decide which models should be chosen. The basic model proposed by Chan-Vese [16] aimed at finding out the global classification centers $c_{1}$ and $c_{2}$, when the classification number is set as 2 . The classification centers of the image are mainly influenced by the total inner and outer region pixels.

Comparing to the inner gray distribution, the segmentation is easily influenced by the gray of the edge pixels. At the same time, because of the inhomogeneous of the region edges, the region edge may exist or not exist in the difference between $c_{1}$ and $c_{2}$. When the edge-grays of the region are all less or higher than the global center $c_{1}$ and $c_{2}$, the global dividing reference may lose its role. Based on Chan-Vese model, local binary fitting energy model [19] (LBF) and local and global intensity fitting energy model [14] (LGIF) considered about the local and global information in the evolution procedure. In order to demonstrate the strengths of the proposed initial contour choosing algorithm, we choose LBF and LGIF as the testing models of the following experiments. Firstly, we compare the proposed initial contour segment results with the other human chosen initial contour. Secondly, we continue with the properties of the proposed initial contour with the other initial contour, and give the comparison of the convergence procedure with LBF and LGIF. Finally, we extend the experiments with various images.

\subsection{The Comparison Different Initial Contour}

Commonly, the accurate choosing of initial contour is an important step in the process of image segmentation. For the purpose of comparing the ability of the proposed model with LBF and LGIF, we choose the different initial contour of the same image. In the experiment, we let the parameter $\sigma$ of Gaussian sampling function as fixed value 4 in all the iteration processing. Figure 5 shows the results of our model and LBF/LGIF model. All the results are after 300 time iteration. Seeing from comparative results of Figure 5, we may found the results of the proposed initial contour is more close to the human visual result than the results of LBF and LGIF. 
Because of the segment region of the testing image has different phase in gray scale, only using the local information or the summarization of the local information may cause the conflict of the two kinds of information. Besides, the LBF model using the local information to segment the image, it heavily depends on the size of the Gaussian sampling parameter $\sigma$. The LGIF model use both the local and global information to segment the image, when the image is two-phased of nearly two-phased, the segment results are close to the proposed initial contour. But this model has a problem in dealing with the local transitional edges.

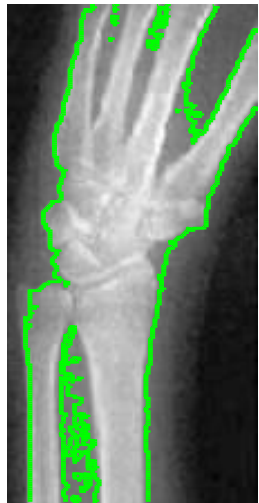

(a)

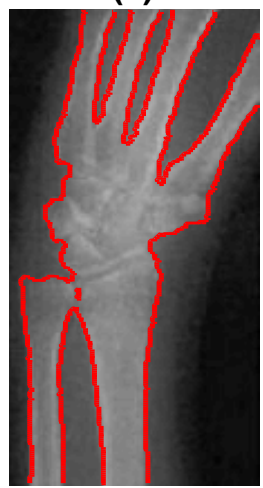

(a1)

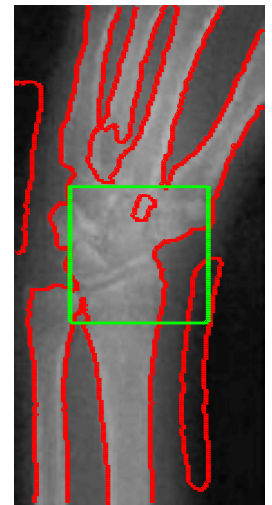

(b)

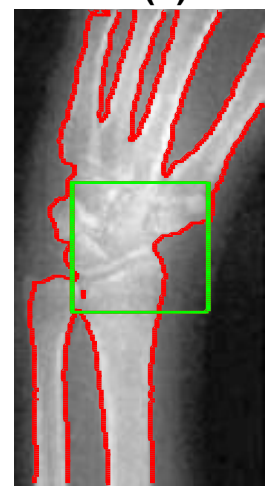

(b1)

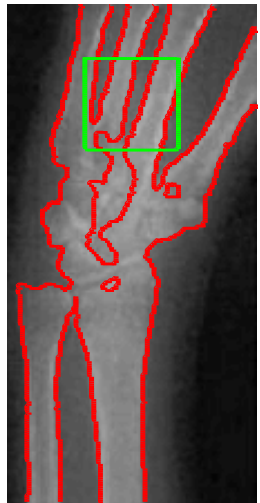

(c)

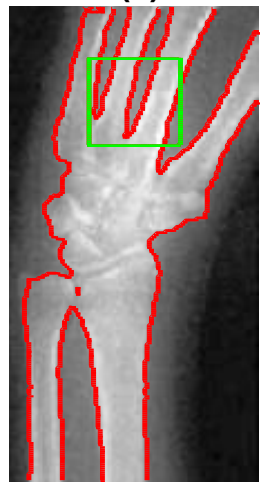

(c1)

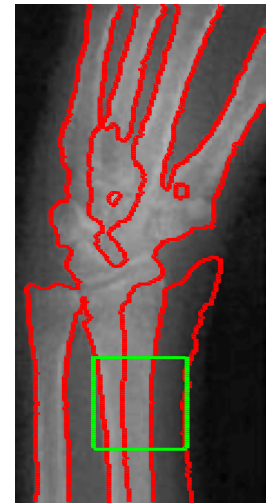

(d)

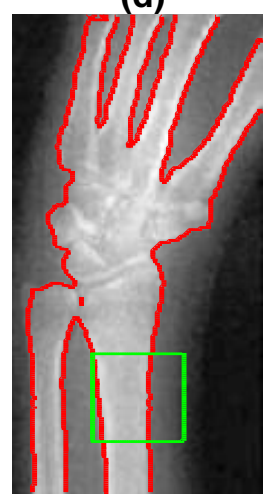

(d1)

Figure 5. The Comparison of the Proposed Initial Contour Algorithm and Human Chosen Contour After $\mathbf{3 0 0}$ Times Iteration, (a) is the Proposed Initial Contour, (a1) is the Iteration Result of LBF with the Initial Contour of (a), (b) -(d) is the Human Chosen Initial Contour and the Iteration Result of LBF. (b1) - (d1) is the Comparative Result of LGIF.

For the purpose of comparing the role of the parameter of Gaussian sampling function, we compare the results of different parameter value. In the experiments, we set the parameter $\sigma$ as 2, 4,6 and 8 in LBF models. From the segmentation results (Figure 6), we found when the parameter is small, the local detail information is emphasized, vice versa, the global information is emphasized. As to the LGIF model, because of the minimization of the energy function is based on the summarization of the local information, the sampling parameter has less influence when the image nearly twophased.

From Figure 6, we may also found, the choosing of parameter is an important problem when using the human chosen initial contour. When the parameter value is bigger, the iteration result is close to the result LGIF except some transitional region edges. Considering of the transitional edges and global dividing conditions, we choose this parameter as the fixed value 4 in all of the following experiments. 


\subsection{The Comparison of the Evolution Efficient}

In this subsection, we give a comparison of our proposed initial contour and the human chosen initial contour in evolution efficient. The different initial contour may lead to different segmentation result (Figure 7), so the comparing of the evolution efficiency becomes very difficult. On the other hand, the segmentation accurate is very difficult to find a uniform standard.

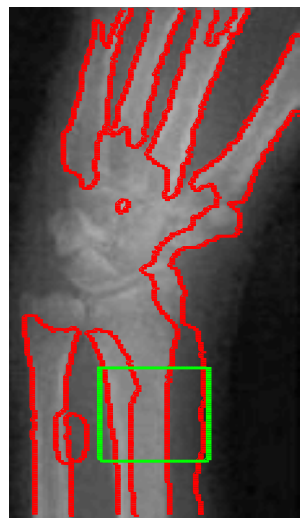

(a) $\sigma=2$

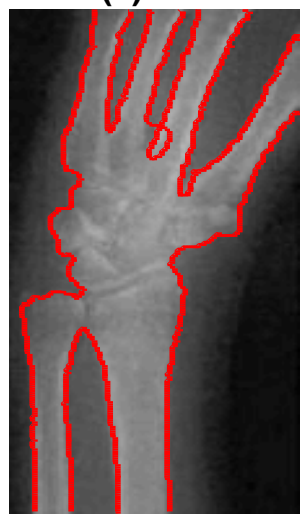

(a1) $\sigma=2$

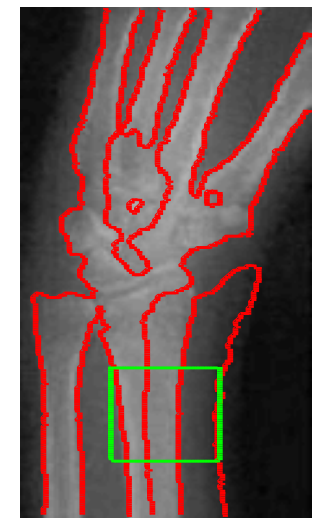

(b) $\sigma=4$

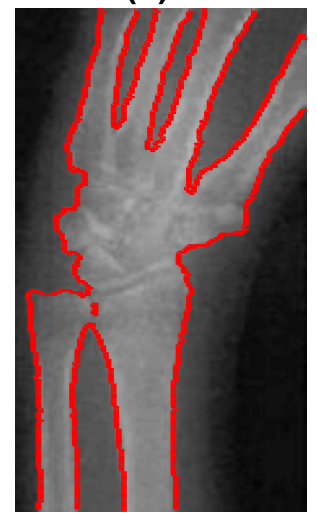

(b1) $\sigma=4$

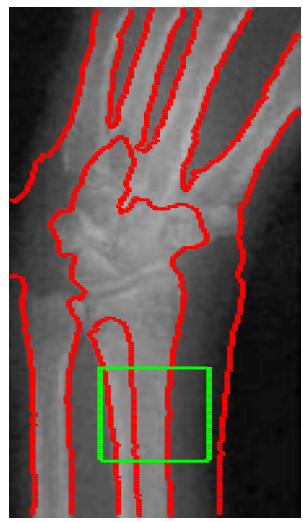

(c) $\sigma=6$

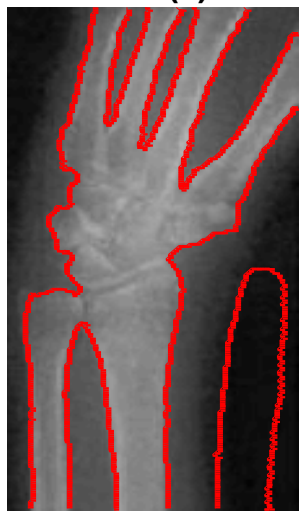

(c1) $\sigma=6$

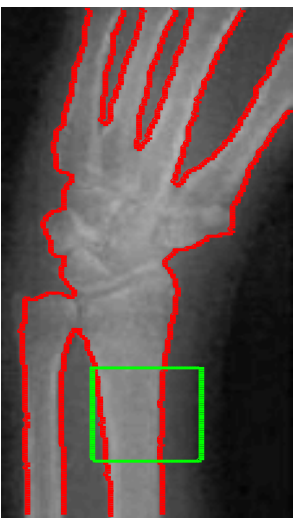

(d) $\sigma=8$

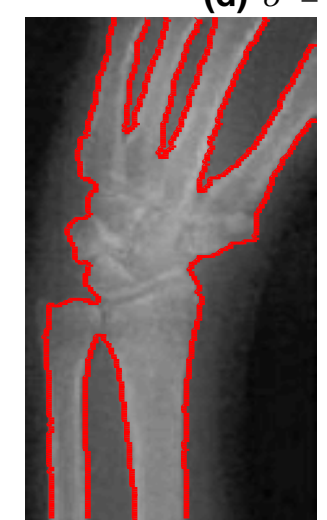

(d1) $\sigma=8$

Figure 6. The Comparison of Different Sampling Value Sigma=2, 4, 6, and 8. The First Row is the Result of the Human Chosen Initial Contour, and the Second Row is the Result of the Initial Contour of Figure 5 (a)

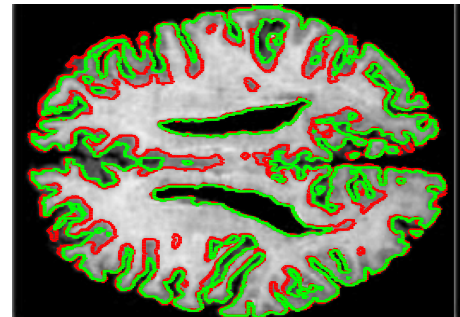

(a)

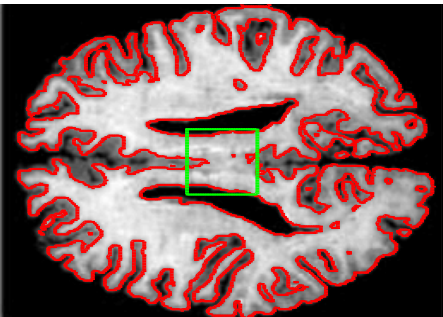

(b)

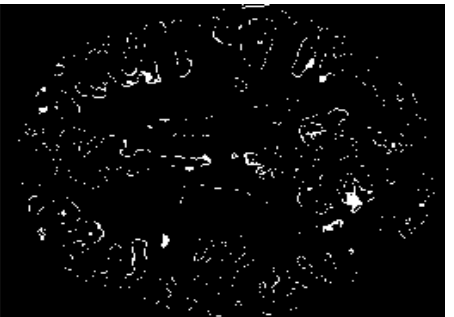

(c)

Figure 7. The Difference of Different Initial Contour with LGIF, (a) is the Segment Result of the Proposed Initial Contour, the Green Lines is the Initial Contour, (b) is the Result of LGIF, and (c) is the Difference of these Two Algorithms

The transitional region is a common phenomenon in image segmentation. It makes the minimization of the energy function of LBF and LGIF sometimes incorrect. So, in the 
comparison of the evolution efficient, we choose the best segmentation parameters artificially and comparing the normalized difference of each iteration. Figure 8 shows the best segmentation results of LBF and LGIF comparing to the convergence procedure of the proposed initial contour based LBF. In Figure 9, we compare the procedure with the same parameter.

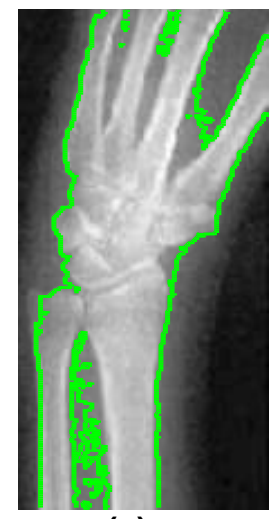

(a)

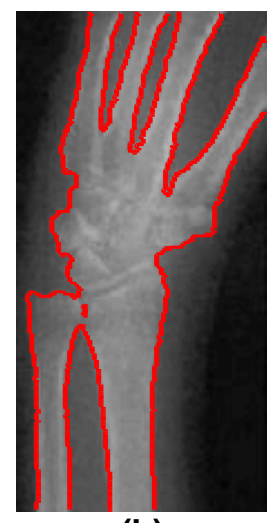

(b)

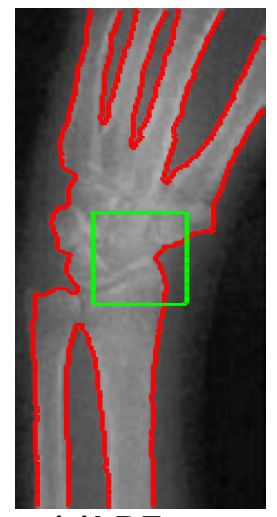

(c)LBF

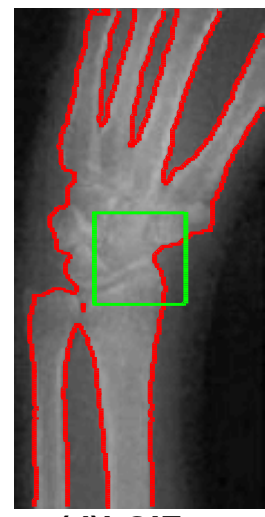

(d)LGIF

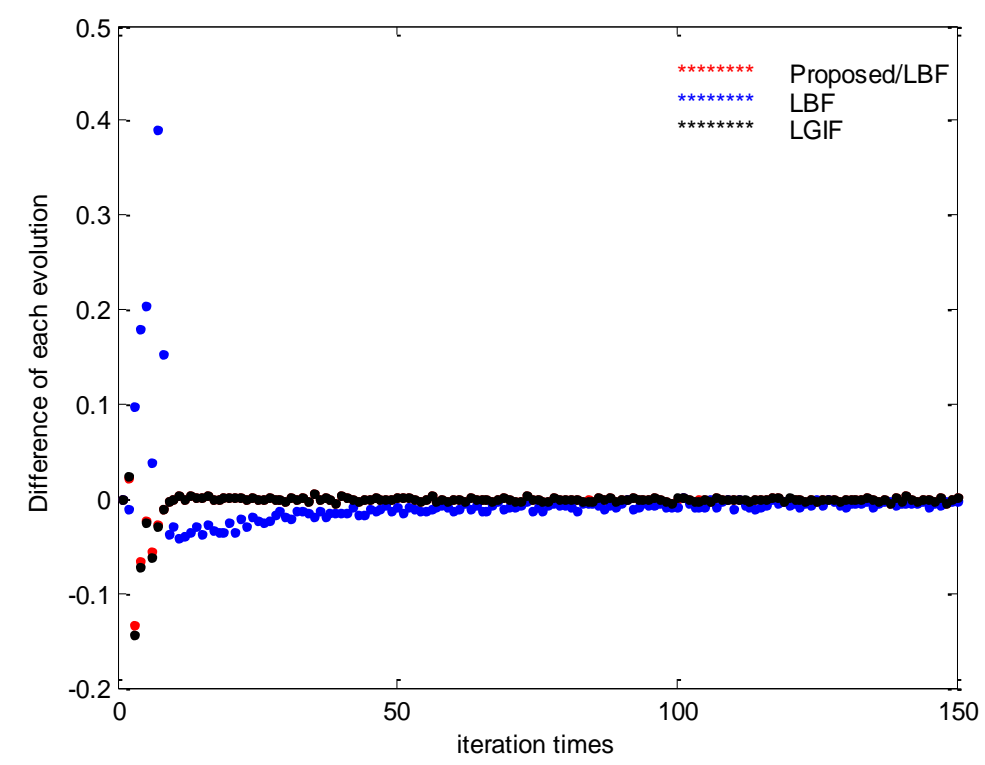

\section{Figure 8. The Comparing of the Convergence Procedure, (a) is the Proposed Initial Contour, (b) is the LBF Result using the Initial Contour of (a), (c) is the LBF Result, (d) is the LGIF Result}

From Figure 8, we found when the parameter is chosen as the best segmentation result, the convergence procedure of the proposed initial contour is close to LGIF procedure. The segmentation result has little difference except some transitional region edges. In Figure 9, on the fixed parameter condition, the convergence procedures have larger difference with each other. In fact, whatever we adjust the parameters, the human chosen initial contour may cause wrong segment results comparing to the result of the proposed initial contour. Seeing from the iteration difference of the experimental images, we may found when the background of the input image is homogeneous or near to the edge difference, the convergence procedure and the results have less difference from each other. When the background is inhomogeneous and the regions are scatter, the convergence results may different from each other, as shown in Figure 9. When the edge image region has more details, the convergence may vibrate in a scale as shown in Figure 9(c). 


\subsection{The Experiments with Different Kinds of Images}

In this part, we will experiments of different images. To validate the adaptability of the proposed automatic initial contour algorithm, we choose the images according to the image region conditions: (1) the single region within the 2-phrase images; (2) the regions are scattered in the images; (3) the region with transitional edges. Figure 10 shows the segmentation result of different images after 100 times iteration. In the experiment, the parameters $\sigma$ and $N$ is set as the fix value 4 and 2 .

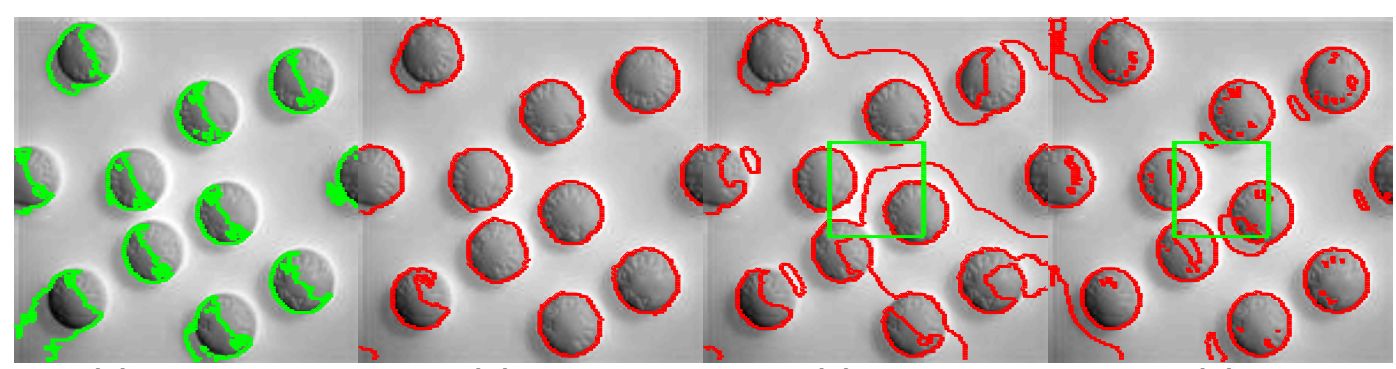

(a)

(b)

(c)LBF

(d)LGIF

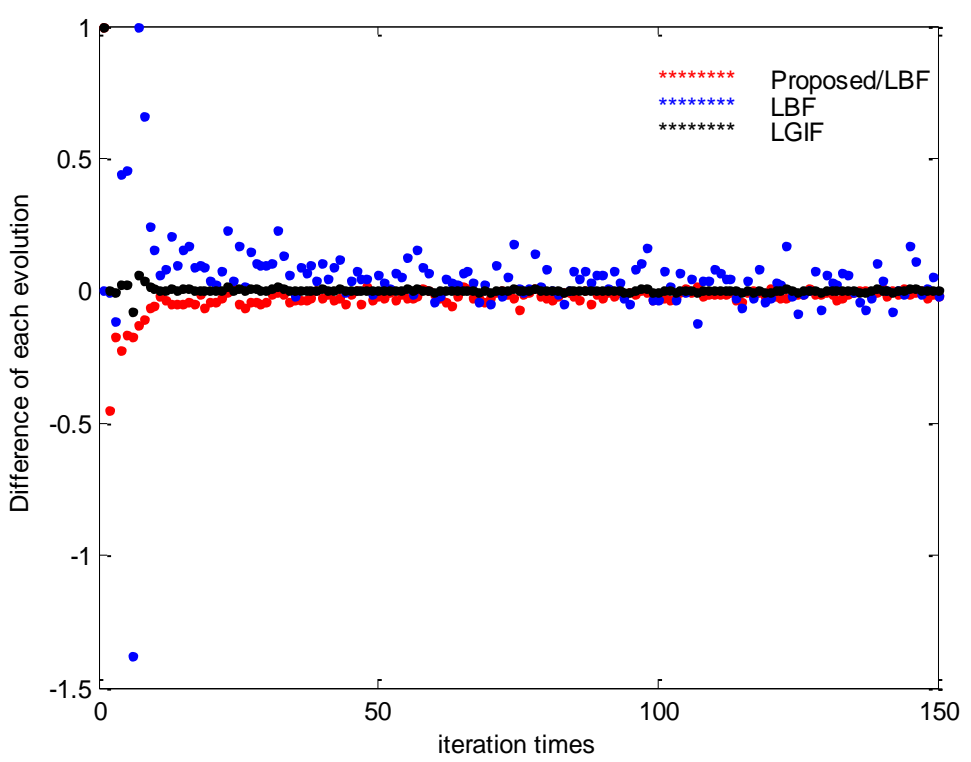

Figure 9. The Comparing of the Convergence Procedure, (a) is the Proposed Initial Contour, (b) is the LBF Result with the Initial Contour of (a), (c) is the LBF Result, (d) is the LGIF Result 


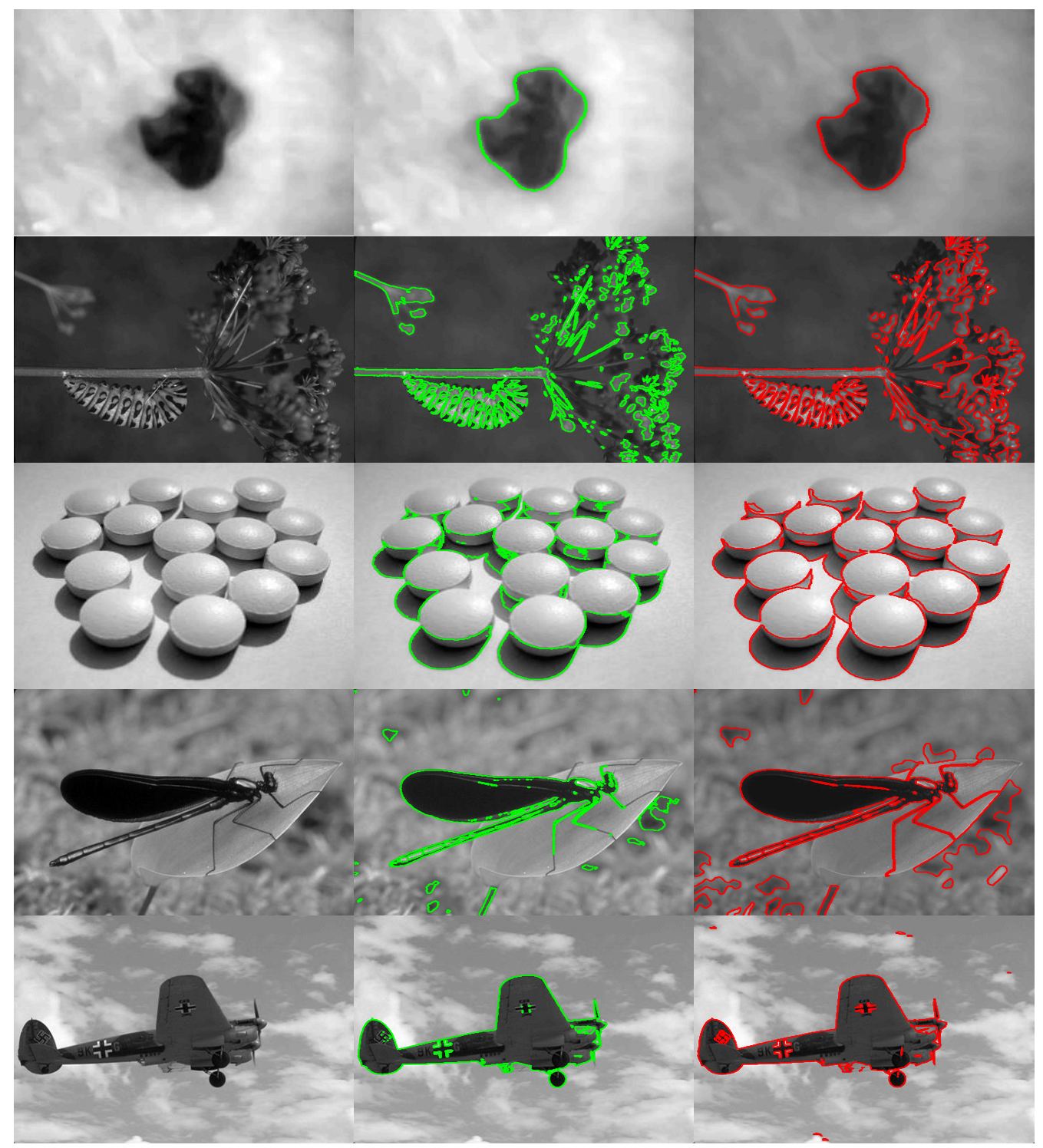

Figure 10. The Proposed Initial Contour and the Segmentation Results of Different Images, the First Column is the Rough Image, the Second Column is the Choosing Initial Contour, the Third Column is the Segment Result

\section{Conclusion}

In this work, we propose a novel automatic initial contour choosing framework based LBF/LGIF for image segmentation. The proposed algorithm in certain case has resulted in significant improvement in accuracy and time consuming. At the same time, this algorithm is adaptable to different images without adjusting the parameters. In our algorithm, the initial contour is chosen by the mean value of the image gray. This dividing may cause some detailed initial contours, when the image foreground and background is very near, the segmentation results may have some little regions. In the following works, we will aim at finding the more stable automatic initial contour choosing algorithm and adjusting the evolution procedure of the energy function especially the consistence of the local information and global information. 


\section{Acknowledgements}

The authors thank the anonymous reviewers for their many valuable comments and suggestions that helped to improve both the technical content and the presentation quality of this paper.

\section{References}

[1] Q. Zheng, E. Dong, Z. Cao, W. Sun and Z. Li, "Active contour model driven by linear speed function for local segmentation with robust initialization and applications in MR brain images," Signal Processing, vol. 97, (2014), pp. 117-133.

[2] W. Wang, L. Zhu, J. Qin, Y.-P. Chui, B. N. Li and P.-A. Heng, "Multiscale geodesic active contours for ultrasound image segmentation using speckle reducing anisotropic diffusion," Optics and Lasers in Engineering, vol. 54, (2014), pp. 105-116.

[3] H. Zhou, J. Zheng and L. Wei, "Texture aware image segmentation using graph cuts and active contours," Pattern Recognition, vol. 46, (2013), pp. 1719-1733.

[4] Q. Zheng and E.-Q. Dong, "Narrow Band Active Contour Model for Local Segmentation of Medical and Texture Images," Acta Astronautica Sinica, vol. 39, (2013), pp. 21-30.

[5] C.-Y. Yu, W.-S. Zhang, Y.-Y. Yu and Y. Li, "A novel active contour model for image segmentation using distance regularization term," Computers \& Mathematics with Applications, vol. 65, (2013), pp. 1746-1759.

[6] X. Xie, J. Wu and M. Jing, "Fast Two-Stage Segmentation via Non-Local Active Contours in Multiscale Texture Feature Space," Pattern Recognition Letters, vol. 34, (2013), pp. 1230-1239.

[7] W. Liu, Y. Shang and X. Yang, "Active contour model driven by local histogram fitting energy," Pattern Recognition Letters, vol. 34, (2013), pp. 655-662.

[8] D. Li, W. Li and Q. Liao, "Active contours driven by local and global probability distributions," Journal of Visual Communication and Image Representation, vol. 24, (2013), pp. 522-533.

[9] Q. Ge, L. Xiao, H. Huang and Z. H. Wei, "An active contour model driven by anisotropic region fitting energy for image segmentation," Digital Signal Processing, vol. 23, (2013), pp. 238-243.

[10] J. Clement, N. Novas, J.-A. Gazquez and F. Manzano-Agugliaro, "An active contour computer algorithm for the classification of cucumbers," Computers and Electronics in Agriculture, vol. 92, (2013), pp. 75-81.

[11] Y. Yuan and C. He, "Adaptive active contours without edges," Mathematical and Computer Modelling, vol. 55, (2012), pp. 1705-1721.

[12] C. He, Y. Wang and Q. Chen, "Active contours driven by weighted region-scalable fitting energy based on local entropy," Signal Processing, vol. 92, (2012), pp. 587-600.

[13] Q. Ge, L. Xiao, H. Huang, and Z. H. Wei, "An active contour model driven by anisotropic region fitting energy for image segmentation," Digital Signal Processing, (2012), pp. 238-243.

[14] L. Wang, C. Li, Q. Sun, D. Xia and C.-Y. Kao, "Active contours driven by local and global intensity fitting energy with application to brain MR image segmentation," Computerized medical imaging and graphics, vol. 33, no. 7, (2009), pp. 520-531.

[15] S. Lankton and A. Tannenbaum, "Localizing region-based active contours," Image Processing, IEEE Transactions on, vol. 17, (2008), pp. 2029-2039.

[16] T. F. Chan and L. A. Vese, "Active contours without edges," Image Processing, IEEE Transactions on vol. 10, (2001), pp. 266-277.

[17] L. Fang and X. Wang, "Image segmentation framework using EdgeFlow-Based active contours," Optik - International Journal for Light and Electron Optics, vol. 124, (2013), pp. 3739-3745.

[18] R. Haralick and L. Shapiro, "Survey: image segmentation techniques," Computer Vision, Graphics and Image Processing, vol. 29, (1985), pp. 100-132.

[19] C. Li, C.-Y. Kao, J. C. Gore and Z. Ding, "Implicit Active Contours Driven by Local Binary Fitting Energy," presented at the 2007 IEEE Conference on Computer Vision and Pattern Recognition, Minneapolis, MN, USA, (2007) June 17-22.

\section{Authors}

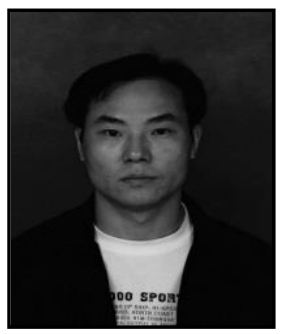

Bo Cai, received his B.S. degree in mechatronics from China Agricultural University, in 1998, and M.S degree in Beijing University of Posts and Telecommunications, in 2003. Currently, he is the Ph.D candidate of China Academy of Engineering Physics. His research interests include image processing and detection. 


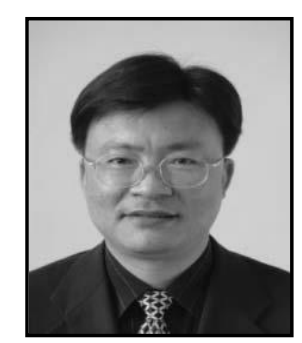

Zhigui Liu, received the Ph.D degree in traffic information engineering and control from Southwest Jiao Tong University, China, in 2006. Currently, he is the teacher of Southwest University of Science \& Technology, and Ph. D tutor of China Academy of Engineering Physics. His research interests include vision sensor technology, image processing and machine vision, etc.

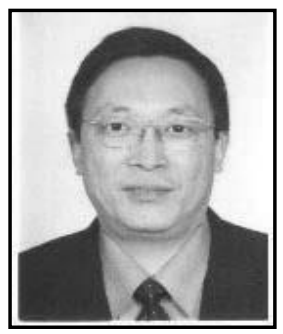

Junbo Wang, received his B.S. degree in physics from Sichuan Normal University, China, in 1981, and the Ph.D degree in optical engineering from Chengdu electronic science and technology University, China, in 1985. Currently, he is the teacher of Southwest University of Science \& Technology, and Ph. D tutor of China Academy of Engineering Physics. His research interests include atmospheric optical and detection, etc.

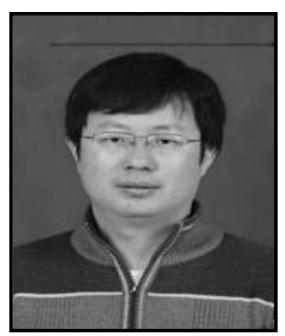

Yuyu Zhu, received his B.S. degree in automation from Southwest University of Science \& Technology, in 2002, and M.S degree in control engineering and control theory, in 2009. Currently, he is the teacher of Southwest University of Science \& Technology. His research interests include signal detection and processing, power designing etc. 